\title{
Preprocessing and Optimization of Smooth Data-driven Model for Emergency Conditions Against Air Pollution
}

\section{Ali Ardalan', H. Mohammadi ${ }^{2 *}$, A. Massah Bavani ${ }^{3}$, Kazem Naddafi ${ }^{4}$, M.T. Talebian ${ }^{5}$}

${ }^{1}$ Department of Disaster and Emergency Health, Iran's National Institute of Health Research; Department of Disaster Public Health, School of Public Health, Tehran University of Medical Sciences, Tehran, Iran;

${ }^{2}$ Department of Disaster Public Health, School of Public Health, Tehran University of Medical Sciences, Tehran, Iran.

${ }^{3}$ Department of Irrigation and Drainage Engineering, College of Abouraihan, University of Tehran.

${ }^{4}$ Department of Environmental Health Engineering, School of Public Health, Tehran University of Medical Sciences, Tehran, Iran; Institute for Environmental Research (IER), Tehran, Iran.

${ }^{5}$ Department of Emergency Medicine, Imam Khomeini Hospital; Tehran University of Medical Sciences, Tehran, Iran.

Study Area: Tehran, Iran

Coordinates: $35^{\circ} 41 \mathrm{~N}$; $51^{\circ} 25^{\prime} \mathrm{E}$

Key words: Air Pollution, Emergency condition, Smooth data, Gamma test, Genetic algorithm

\section{Introduction:}

DIt is well known that air quality and climate are expected to have an impact up to far extent on the human health (Bernard, Samet et al., 2001, Brunekreef \& Holgate 2002, Anderson 2009, Grell \& Baklanov 2011, Vanos et al., 2015). Air pollution could be categorised as extreme weather events belonging to environmental degradation/ disasters. The most notable disaster due to air pollution was from Meuse Valley, the year 1930 (Nemery et al., 2001). Further, studies from 1939- Saint Louis, 1948- Donora, 1952- London, 1983- Melbourne, 1973- Los Angles, 1997Indonesia, 2005- Malaysia, and 2006- Southeast Asia (IITM, 2015) have mentioned them as uncontrolled confounders for analysing such events. Such uncontrolled

\section{Abstract}

Magnitudes of the air pollution depend on various variables. Preprocessing and optimisation processes are necessary to discover the complexity of the relationship of the data for more accurate and efficient predictions. These techniques help to clean the datasets and to find the best structure of the smooth data model. The Gamma test (GT) and Genetic Algorithm (GA) are practical tools which can be applied for preprocessing and optimising data models. Regarding building a smooth data model, the developed artificial neural networks are functional optimisation strategies which are suitable for ANN training. Moreover, local linear regression (LLR) and dynamic local linear regression (DLLR) models are effective due to the high density of our normalised dataset. In this regard, we developed a process to construct a smooth data model to support environmental decision making in air pollution emergency conditions. The main objective of this work was to set an appropriate algorithm by preprocessing and optimising a set of the data model for developing smooth data-driven models which could play a significant role in early warning systems in regard to the human health. The data sets included the meteorological and air pollutant variables as inputs/predictors and emergency medical service clients as outputs. The GT and GA were applied to analyse and optimise the input variables. Three types of ANNS (ANN1, $\mathrm{ANN}_{2}$, and $\mathrm{ANN}_{3}$ ), (LLR), and (DLLR) techniques were used to establish the models. Finally, a smooth data model was constructed and evaluated.

variables are available everywhere, and can be analysed for forecasting and prediction of future climatic conditions. Since 1990, air quality modelling has been considered the most effective tool for the assessment of harmful impacts and risk of air pollution (Zannetti, 2013). Several studies have been conducted to determine the interaction of meteorological parameters and air pollutants and their impact on human health consequences. In this context, most air pollution models applied in various applications include proximity models, interpolation models, Landuse regression models (Hoek et al., 2008), dispersion models, integrated meteorological-emission models, hybrid models, (Jerrett et al., 2004), multiple linear regression, principal components analysis, empirical 
orthogonal function analysis, cluster analysis, stochastic, fine resolution atmospheres, multi-pollutant exchange, and artificial neural networks (ANNs). Sophisticated statistical methods have been employed by various environmental epidemiologists for analysing effects of air pollution on the human health consequences (Bernard et al., 2001; Elliott et al., 2007; Gasparrini, 2011; Sof ianopoulou et al., 2013; Vanos et al., 2015).

A number of linear and nonlinear methods have been applied to time series for forecasting the concentration of air pollutants such as $\mathrm{O}_{3}, \mathrm{PM}, \mathrm{NO}_{2}, \mathrm{CO}$, and $\mathrm{SO}_{2}$ while compared to ANNs (Perez \& Gramsch, 200o; Solaiman et al., 2008; Moustris et al., 2009; Inal, 2010; de Gennaro, et al., 2013).

ANNs are useful tools for forecasting air pollution conditions and providing better results as compared to the other relevant models (Gardner \& Dorling, 1998).

The comparison of some predictive modelling algorithms indicated that the quality of the results depended on the quality of the data set, the interaction between predictors Vs target parameters, and the evaluation criteria. ANNs have some advantages such as less formal statistical training, the capability to detect nonlinear relationships between dependent and independent variables, all possible interactions between predictor and independent variables, and the availability of multiple training algorithms. However, ANNs are affected by limitations such as the "black box" nature, more computational burden, proneness to over-fitting, and empirical nature of model development.

Genetic algorithm (GA) can be used to eliminate the ANN overtraining phenomenon due to optimisation of the input variables, transfer functions, and ANN structure (Bibi et al., 2002; Liao et al., 2012; Pires et al., 2012). GA also is a search tool loosely connected to artificial intelligence (Holland 1975; Demuth \& Beale 2002; Goyal et al., 2006; Malhotra et al., 2011; Liao et al., 2012).

The Gamma test (GT) and GA recognize the most important input to predict the target's outputs using intelligent non-linear modeling, and to identify how much data is sufficient to form a smooth data model, how liable the model is, and how to build appropriate smooth data-driven models for forecasting and predicting health consequences in air pollution episodes (Jones, 1998; Durrant, 2001).

The M-test is the other test which indicates how the GT estimate changes as more data are used for its computing. This test can also determine how much data is possibly required to have a model of a given quality. By performing this test, we can find whether there was suff icient data to obtain a reliable gamma estimate(Noori, et al., 2011; Asagha et al., 2014). In the modelling processes, the selection of the most important and relevant predictors is essential. The predictors can be selected on the basis of linear and partial autocorrelation analyses and nonlinear sensitivity analyses (Solaiman etal., 2009).

Studies have shown that air quality relies on meteorological conditions and the correlation of weather variables and air pollutants is poorly understood or is seen uncertain. Due to its extreme complexity and the very strong nonlinear characteristics of forecasting air pollution, using accurate and quantitative data models is necessary and can help us with simulation and modelling regarding the prediction of the future health impact of these events. The limiting factors of the predictive accuracy of the models are the presence of measurement noise and insuff icient data.

This paper presents a new approach to developing an algorithm for preprocessing and optimising the data model in order to choose the best combination of meteorological variables and air pollutants with the most accurate and efficient fit and clean data to estimate the human health risk. Furthermore, we considered the sufficient amount of data for modelling and we also calculated the threshold limit value to perform the model. Finally, five types of smooth data models were established and evaluated.

\section{Materials and methods:}

Study area \& data collection: this study was conducted in Tehran, the capital of Iran which is populated by $\sim 9$ million inhabitants. It is located at an altitude of 1000180o. Tehran features a semi-arid climatic conditions having various environmental critical issues, so that about 5521 out of all 47284 mortality cases in 2010 were due to short term effects of air pollution (Atash, 2007; Naddafi et al., 2012).

In this study the daily average and 3-day moving average of meteorological and air pollutant data, as inputs, was used (Table 1 ).

The number of cardiovascular and respiratory EMS clients were selected as output/target in the current day and $1,3,5$ and 7 days after.

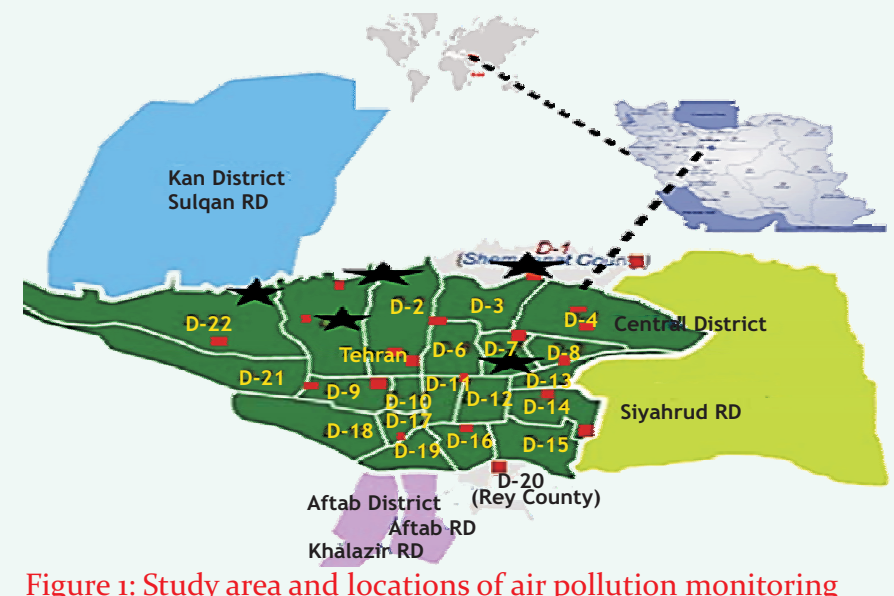

Figure 1: Study area and locations of air pollution monitoring and synoptic stations 
Table1: Twenty five input variables used as initial inputs at dataset during 2011-2013 (1096 days)

\begin{tabular}{|c|c|c|}
\hline & Variables & Scale \\
\hline \multicolumn{3}{|c|}{ Meteorological parameters } \\
\hline 1 & Daily Maximum temperature (Tmax.) & $\mathrm{oC}$ \\
\hline 2 & 3-day moving average of TMax. & $\mathrm{oC}$ \\
\hline 3 & Daily Minimum temperature (TMin.) & $\mathrm{oC}$ \\
\hline 4 & 3-day moving average of TMin. & $\mathrm{oC}$ \\
\hline 5 & Daily Precipitation (P) & $\mathrm{Mm}$ \\
\hline 6 & 3-day moving average $\mathrm{P}$ & $\mathrm{Mm}$ \\
\hline 7 & Wind speed (WS) & $\mathrm{m} / \mathrm{s}$ \\
\hline 8 & 3-day moving average WS & $\mathrm{m} / \mathrm{s}$ \\
\hline 9 & Relative humidity (RH) & $\%$ \\
\hline 10 & 3-day moving average $\mathrm{RH}$ & $\%$ \\
\hline \multicolumn{3}{|c|}{ Air Pollutants } \\
\hline 11 & Carbon monoxide (CO) & $\mathrm{AQI}$ for $\mathrm{CO}$ \\
\hline 12 & 3-day moving average $\mathrm{CO}$ & $\mathrm{AQ}[$ for $\mathrm{CO}$ \\
\hline 13 & Ground- level Ozone $\left(\mathrm{O}_{3}\right)$ & $\mathrm{AQI}$ for $\mathrm{O}_{3}$ \\
\hline 14 & 3 days moving average $\mathrm{O}_{3}$ & $\mathrm{AQI}$ for $\mathrm{O}_{3}$ \\
\hline 15 & Nitrogen dioxide $\left(\mathrm{NO}_{2}\right)$ & $\mathrm{AQI}$ for $\mathrm{NO}_{2}$ \\
\hline 16 & 3-day moving average $\mathrm{NO}_{2}$ & AQIfor $\mathrm{NO}_{2}$ \\
\hline 17 & Sulf ide dioxide (Soz) & AQI for So2 \\
\hline 18 & 3-day moving average Soz & AQI for So2 \\
\hline 19 & $\begin{array}{l}\text { Particulate Matter less } 10 \text { micrometers } \\
\text { in diameter (PMio) }\end{array}$ & AQI for Pmio \\
\hline 20 & 3-day moving average PMio & AQI for PMio \\
\hline 21 & $\begin{array}{l}\text { Particulate Matter less } 2.5 \text { micrometers } \\
\text { in diameter (PM2.5) }\end{array}$ & AQI for PM2.5 \\
\hline 22 & 3-day moving average PM2.5 & AQI for PM2.5 \\
\hline 23 & Air quality index ( AQI) & AQI \\
\hline 24 & 3-day moving average AQI & AQI \\
\hline 25 & Stability class (SC) ( dt/dz) per 10om* & /10om \\
\hline
\end{tabular}

search options of GA to find good embedding were the population size of 100 chromosomes, the mutation rate of 0.01, the crossover rate of 0.5 , the gradient fitness of o.1, intercept fitness of 0.8 , and length fitness of o.1. The software package used to analyze the data was win Gamma. It is a non-linear analysis and modeling tool developed by the Department of Computer Science, Cardiff University (Jones, 1998).

Most of the input parameters in the dataset were incompatible (e.g. temperature, wind speed, etc.). In order to ensure that all variables at lease started with an equal chance to contribute to an output prediction, standard normalization was applied. Then, we built and examined various smooth data modeling techniques. In the first step, Three principle factors of Gamma test which were applied to determine the fitness of particular mask or feature set were Gamma statistics (the intercept), model complexity (the gradient), and the number of inputs required (the length).

In the next step, following three smooth data-driven modeling techniques, as modular prediction systems, were constructed and examined. Artificial Neural Network (ANN) as an error minimization techniques for training algorithm method, Local linear regression(LLR) and Dynamic local linear regression (DLLR).

The three types of ANN include two layers back propagation ( $\left.A N_{1}\right)$, The conjugate gradient training algorithm as an alternative function optimization $\left(\mathrm{ANN}_{2}\right)$ and (BFGS)Broyden-Fletcher-Goldfarb-Shanno ( $\left.\mathrm{ANN}_{3}\right)$. Regarding model identification, using the win Gamma software, the EMS clients' prediction model was built by the $\mathrm{ANN}_{1}$ which contained two layer backpropagation

In this research, the GT, GA and Mtest were performed to preprocess and optimize the data model. We used these tests to find the model complexity, the quality of the data, the stopping criteria for training (by M- test), and the best model performance prior to model building. We determined the noise present in the dataset prior to model building and also, whether there was sufficient data to build an appropriate model and the significance of the variables (by GT). In addition, it was applied to reduce or eliminate the need for a separatevalidation set.

We used GA to identify the appropriate combination of the candidate inputs (meteorological and pollutant variables) which were most

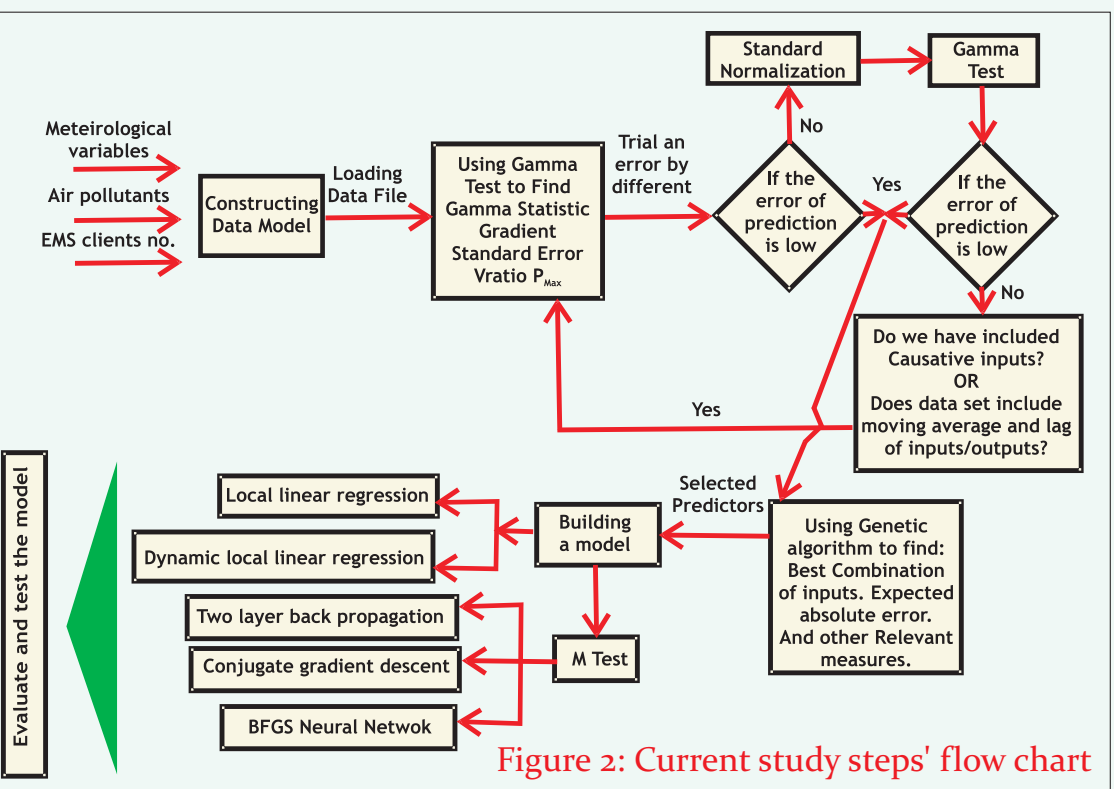
likely to influence the prediction of EMS clients with cardiovascular and respiratory problems. In training algorithms. We evaluated ANN1 with various this research, the selected parameters for controlling the numbers of nodes in the first and second layer. 
Constructing and testing $\mathrm{ANN}_{3}$ based on the BFGS training algorithm was done with various numbers of nodes in the first and second layer of two layers back propagation.

\section{Results}

Table- 2 shows the Gamma test results including Gamma statistics and other relevant measures for 25 inputs and 10 outputs which were all used in the first analysis.

Gamma statistic is calculated by determining a regression line and is actually the vertical intercept of the regression line. It is the estimated variance of errors for any smooth data model. It is used for calculating the expected absolute error. It means that any smooth data built on this dataset will have a standard deviation of prediction error to evaluate the outputs. The gradient is the slope of regression line and standard error (SE) is the usual goodness of fit applied to the regression line. SE indicates the confidence of the estimated noise variance on the whole 10 outputs in the value of gamma. The $V$ ratio is def ined as Gamma/var. (output). The $\mathrm{V}$ ratio close to o shows a high degree of predictable by a smooth data model.

Regarding the size of the dataset, near neighbors (number of Pmax) was selected. The variation of Gamma statistic and the standard error versus the number of Pmax has been shown in Fig.3. It can be seen that Pmax=10 is likely to produce the most accurate estimate.

To determine whether there was suff icient data to build a smooth data model, the M- test was applied. This test showed that the adequate model could be built by using 550- 650 data points (Fig.4). It represented that 550650 points were sufficient in constructing a smooth data model without overtraining. Therefore, 600 data points were considered as part of the data for training in ANNs.
To find the best combination of input variables for predicting each target, the GA search and optimization techniques were applied. The abovementioned intercept fitness, gradient fitness, and length fitness were selected for better control of the GA search. A good embedding that can be applied to construct a successful model or simulation can be achieved by capturing the systematic behavior of inputs. An embedding is designated by a string of " 1 "sand "o"s known as a mask. The selected mask (" 1 ") from 25 inputs for each output is shown in Table 3.The arrangement of sort entries in Table 3 is similar to Table 1.

The selected inputs with the best Gamma statistic \& other relevant measurements are presented in Table 4.

As a sample, the scatter plot and $3 \mathrm{D}$-histogram for the selected combination of inputs and o days after EMS cardiovascular clients have been shown in Figure 5 and 6 . The results indicated that model construction with our dataset was feasible.

After analyzing the data with the Gamma test, we evaluated which embedding of the input was suitable and how much data was suff icient.

In this regard, three types of ANNs ( $\left.\mathrm{ANN}_{1}, \mathrm{ANN}_{2}, \mathrm{ANN}_{3}\right)$, LLR and DLLR techniques were used to establish the models.

It should be noted that since the model construction and testing process were massive, we summarized their results and the best implications in the given tables and figures.

The best experiments and their performance of $A N N_{1}$, including statistical values of training and validation phase with the 16 above mentioned selected inputs for each 10 outputs are presented in Table 5. The ANN2f inal results are summarized in Table 6 and Table 7 indicates the $\mathrm{ANN}_{3}$ results.

Table- 2: Gamma test results for scaled dataset

\begin{tabular}{|c|c|c|c|c|c|c|c|c|c|c|}
\hline Evaluate output & EMS & rdiova & lar clie & & & & S Respi & ory cli & & \\
\hline Day(s) after & 0 & 1 & 3 & 5 & 7 & $\mathrm{o}$ & 1 & 3 & 5 & 7 \\
\hline Gamma statistic & 0.033 & 0.019 & 0.028 & 0.030 & 0.027 & & 0.125 & 0.136 & 0.118 & 0.127 \\
\hline Expected Absolute Error & 0.1817 & 0.138 & 0.168 & 0.172 & 0.166 & 0.353 & 0.364 & 0.369 & 0.343 & 0.357 \\
\hline Gradient & 0.0492 & 0.055 & 0.052 & 0.050 & 0.050 & 0.026 & 0.0157 & 0.015 & 0.022 & 0.0154 \\
\hline Standard Error (SE) & 0.004 & 0.008 & 0.005 & 0.007 & 0.003 & 0.008 & 0.005 & 0.009 & 0.004 & 0.006 \\
\hline R Square & 0.87 & 0.92 & 0.89 & 0.88 & 0.89 & 0.50 & 0.47 & 0.45 & 0.53 & 0.49 \\
\hline V Ratio & 0.132 & 0.076 & 0.113 & 0.019 & 0.110 & 0.49 & 0.532 & 0.54 & 0.47 & 0.500 \\
\hline
\end{tabular}

\begin{tabular}{|cc|}
\hline \multicolumn{2}{|c|}{ Table 3: Embedding of inputs chosen from 25 inputs } \\
\hline EMS Cardiovascular clients & Currant day \\
EMS Respiratory clients & 111110111010101010110010 \\
\hline
\end{tabular}

Table 4: GA search Results for finding the best embedding of inputs (16/25 inputs)

\begin{tabular}{|lllllllllll|}
\hline Evaluate output & \multicolumn{4}{l}{ EMS Cardiovascular clients } & \multicolumn{5}{c|}{ EMS Respiratory clients } \\
\hline Day(s) after & 0 & 1 & 3 & 5 & 7 & 0 & 1 & 3 & 5 & 7 \\
\hline Gamma statistic & 0.033 & 0.019 & 0.028 & 0.030 & 0.027 & & 0.125 & 0.136 & 0.118 & 0.127 \\
Gamma & 0.0144 & 0.017 & 0.017 & 0.022 & 0.024 & 0.100 & 0.121 & 0.121 & 0.116 & 0.098 \\
\hline
\end{tabular}

Ambient Science (2016) 


\begin{tabular}{|lllllllllll|}
\hline Expected AbsoluteError & 0.120 & 0.132 & 0.132 & 0.147 & 0.15 & 0.296 & 0.348 & 0.348 & 0.340 & 0.314 \\
Gradient & 0.121 & 0.119 & 0.121 & 0.11 & 0.11 & 0.076 & 0.046 & 0.046 & 0.047 & 0.072 \\
Standard Error & 0.0033 & 0.006 & 0.005 & 0.005 & 0.005 & 0.007 & 0.008 & 0.007 & 0.007 & 0.005 \\
R Square & 0.94 & 0.931 & 0.93 & 0.91 & 0.90 & 0.599 & 0.516 & 0.516 & 0.537 & 0.605 \\
V Ratio & 0.058 & 0.069 & 0.069 & 0.087 & 0.096 & 0.400 & 0.480 & 0.480 & 0.46 & 0.39 \\
Nearest neighbors & 10 & 10 & 10 & 10 & 10 & 10 & 10 & 10 & 10 & 10 \\
\hline
\end{tabular}

Table 5: Results of ANN1 training and testing phases

\begin{tabular}{|c|c|c|c|c|c|c|c|c|c|c|}
\hline Evaluate output & EMS C & iovas & clients & EMS Re & piratol & lients & & & & \\
\hline Day(s) after & $\mathrm{O}$ & 1 & 3 & 5 & 7 & $\mathrm{O}$ & 1 & 3 & 5 & 7 \\
\hline Target MSE & 0.098 & 0.32 & 0.166 & 0.0852 & 0.09 & 0.068 & 0.05 & 0.09 & 0.23 & 0.062 \\
\hline MSE reached & 0.079 & 0.22 & 0.164 & 0.0850 & 0.09 & 0.14 & 0.18 & 0.14 & 0.23 & 0.151 \\
\hline Learning rate & 0.25 & 0.25 & 0.25 & 0.25 & 0.25 & 0.25 & 0.25 & 0.25 & 0.25 & 0.25 \\
\hline Momentum & 0.1 & 0.1 & 0.1 & 0.1 & 0.1 & 0.1 & 0.1 & 0.1 & 0.1 & 0.1 \\
\hline Regularization & ${ }_{1} \mathrm{E}-7$ & 1 E-7 & ${ }_{1} \mathrm{E}-7$ & ${ }_{1} \mathrm{E}-7$ & ${ }_{1} \mathrm{E}-7$ & ${ }_{1} \mathrm{E}-7$ & $1 \mathrm{E}-7$ & $1 \mathrm{E}-7$ & ${ }_{1} \mathrm{E}-7$ & $1 \mathrm{E}-7$ \\
\hline No. of node in first layer & 8 & 8 & 8 & 8 & 8 & 8 & 8 & 8 & 8 & 8 \\
\hline No. of node in second layer & 8 & 8 & 8 & 8 & 8 & 8 & 8 & 8 & 8 & 8 \\
\hline Testing MSE & 0.10 & 0.27 & 0.18 & 0.11 & 0.12 & 0.17 & 0.21 & 0.19 & 0.26 & 0.17 \\
\hline
\end{tabular}

Table 6: Results of ANN2

\begin{tabular}{|c|c|c|c|c|c|c|c|c|c|c|}
\hline Evaluate output & EMS C & iovas & clients & EMS R & pirato & ients & & & & \\
\hline Day(s) after & $\mathrm{O}$ & 1 & 3 & 5 & 7 & 0 & 1 & 3 & 5 & 7 \\
\hline Target MSE & 0.098 & 0.32 & 0.16 & 0.085 & 0.94 & 0.068 & 0.10 & 0.097 & 0.23 & 0.63 \\
\hline Training MSE reached & 0.097 & 0.18 & 0.16 & 0.085 & 0.94 & 0.099 & 0.12 & 0.12 & 0.2 & 0.23 \\
\hline No. of node in first layer & 5 & 5 & 5 & 5 & 5 & 5 & 5 & 5 & 5 & 5 \\
\hline No. of node in second layer & 5 & 5 & 5 & 5 & 5 & 5 & 5 & 5 & 5 & 5 \\
\hline Testing MSE & 0.13 & 0.22 & 0.16 & 0.1 & 0.11 & 0.198 & 0.17 & 0.18 & 0.25 & 0.23 \\
\hline
\end{tabular}

Table 7: Results of $\mathrm{ANN}_{3}$

\begin{tabular}{|c|c|c|c|c|c|c|c|c|c|c|}
\hline \multirow{2}{*}{$\begin{array}{l}\text { Evaluate output } \\
\text { Day(s) after }\end{array}$} & \multicolumn{4}{|c|}{ EMS Cardiıo96ovascular clients } & \multicolumn{6}{|c|}{ EMS Respiratory clients } \\
\hline & $\mathrm{o}$ & 1 & 3 & 5 & 7 & $\mathrm{o}$ & 1 & 3 & 5 & 7 \\
\hline Target MSE & 0.098 & 0.32 & 0.16 & 0.085 & 0.094 & 0.068 & 0.05 & 0.09 & 0.23 & 0.06 \\
\hline Training MSE reached & 0.097 & 0.25 & 0.15 & 0.084 & 0.094 & 0.068 & 0.05 & 0.09 & 0.18 & 0.06 \\
\hline No. of node in first layer & 7 & 7 & 7 & 7 & 7 & 7 & 7 & 7 & 7 & 7 \\
\hline No. of node in second layer & 7 & 7 & 7 & 7 & 7 & 7 & 7 & 7 & 7 & 7 \\
\hline Testing MSE & 0.11 & 0.28 & 0.19 & 0.11 & 0.11 & 0.20 & 0.25 & 0.18 & 0.20 & 0.26 \\
\hline
\end{tabular}

Table8: Results of the LLR model

\begin{tabular}{|lllllllllll|}
\hline Evaluate output & \multicolumn{2}{l|}{ EMS Cardiovascular clients } & \multicolumn{2}{l|}{ EMS Respiratory clients } \\
\hline Day(s) after & $\mathrm{O}$ & 1 & 3 & 5 & 7 & 0 & 1 & 3 & 5 \\
\hline No. of nearest neighborhood & 10 & 10 & 10 & 10 & 10 & 10 & 10 & 10 & 10 & 10 \\
Tolerance & $1 \mathrm{E}-6$ & $1 \mathrm{E}-6$ & $1 \mathrm{E}-6$ & $1 \mathrm{E}-6$ & $1 \mathrm{E}-6$ & $1 \mathrm{E}-6$ & $1 \mathrm{E}-6$ & $1 \mathrm{E}-6$ & $1 \mathrm{E}-6$ & $1 \mathrm{E}-6$ \\
MSE & 4.35 & 4.03 & 4.11 & 3.56 & 3.88 & 7.26 & 8.03 & 1.02 & 7.23 & 6.95 \\
& $\mathrm{E}-30$ & $\mathrm{E}-30$ & $\mathrm{E}-30$ & $\mathrm{E}-30$ & $\mathrm{E}-30$ & $\mathrm{E}-30$ & $\mathrm{E}-30$ & $\mathrm{E}-29$ & $\mathrm{E}-30$ & $\mathrm{E}-30$ \\
\hline
\end{tabular}

Table 9: Results of the DLLR model

\begin{tabular}{|c|c|c|c|c|c|c|c|c|c|c|}
\hline \multirow{2}{*}{$\begin{array}{l}\text { Evaluate output } \\
\text { Day(s) after }\end{array}$} & \multicolumn{3}{|c|}{ EMS Cardiovascular clients } & \multicolumn{7}{|c|}{ EMS Respiratory clients } \\
\hline & 0 & 1 & 3 & 5 & 7 & 0 & 1 & 3 & 5 & 7 \\
\hline \multirow{4}{*}{$\begin{array}{l}\text { No. of nearest neighborhood } \\
\text { Tolerance } \\
\text { MSE }\end{array}$} & 10 & 10 & 10 & 10 & 10 & 10 & 10 & 10 & 10 & 10 \\
\hline & 1 E-6 & 1 E-6 & $1 \mathrm{E}-6$ & 1 E-6 & 1 E-6 & $1 \mathrm{E}-6$ & $1 E-6$ & 1 E-6 & 1 E-6 & ${ }_{1} \mathrm{E}-6$ \\
\hline & 7.84 & 9.78 & 1.12 & 9.57 & 8.84 & 2.12 & 2.24 & 2.53 & 1.71 & 1.61 \\
\hline & E-30 & E-30 & E-29 & E-30 & E-30 & E-29 & E-29 & E-29 & E-29 & E-29 \\
\hline
\end{tabular}




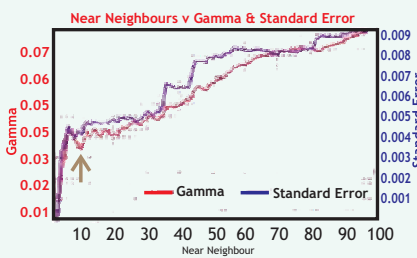

Figure: 3- Variation of gamma and SE as the number of near neighbors

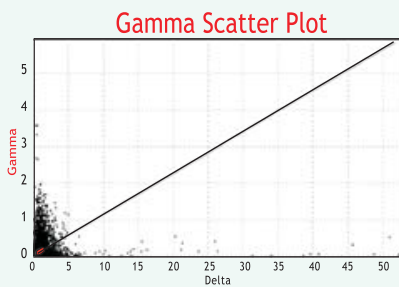

Figure: 5- Scatter plot for cardiovascular clients o day after
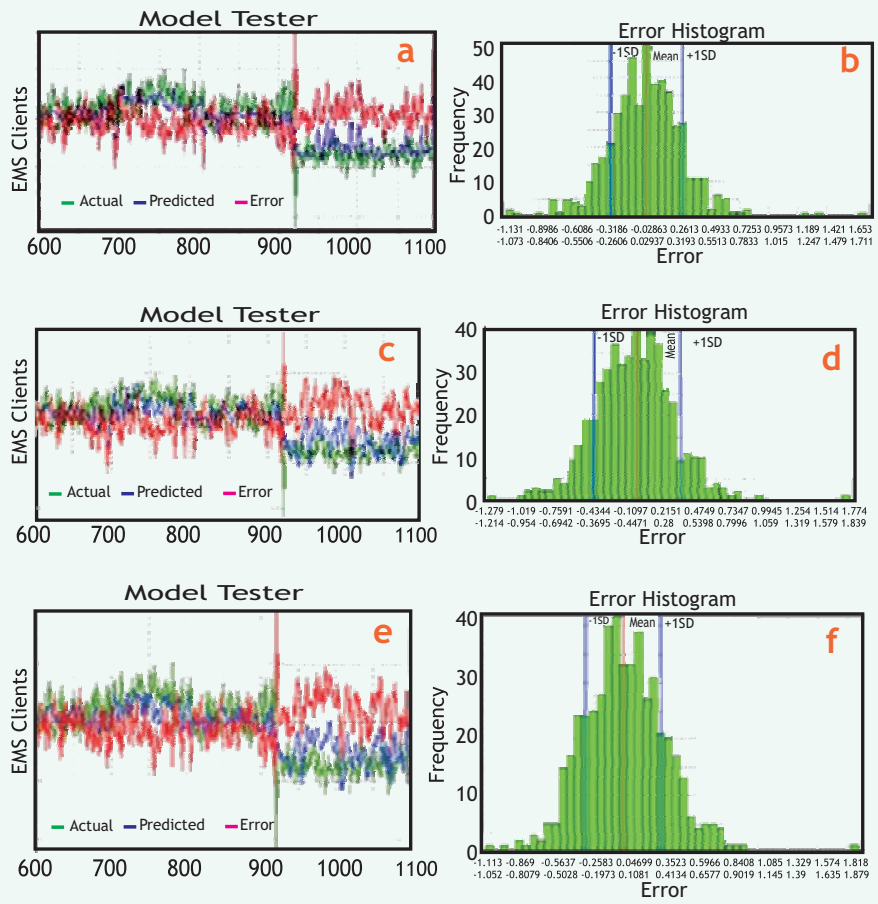

Figure: 7- ANN model tester for EMS cardiovascular clients o day after: a-ANN1, c- ANN2, e- $\mathrm{ANN}_{3}$

Figure: 8- $\mathrm{ANN}_{3}$ error histogram for EMS cardiovascular clients o day after: b-ANN1, d- $\mathrm{ANN}_{2}$, f-ANN 3

\section{Discussion:}

Several studies have applied ANNs to forecast and predict the concentration of one or more air pollutants in an area, as well as its effects on the human health consequences (Gardner \& Dorling, 1998; Grivas \& Chaloulakou, 2006; Liao et al., 2012, Pires et al., 2012). In the present study, three types of two-layer back propagation ANNs with different training algorithms, LLR and DLLR, were applied for data modeling. The structure of the models is shown in

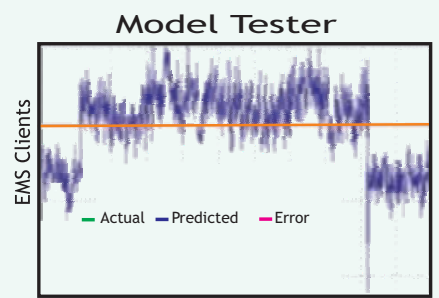

1002003004005006007008009001000

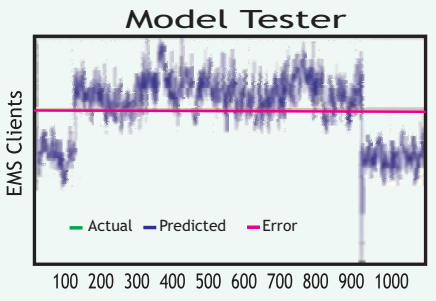

Figure: 9- model tester for EMS cardiovascular clients o day after: a-LLR, c-DLLR
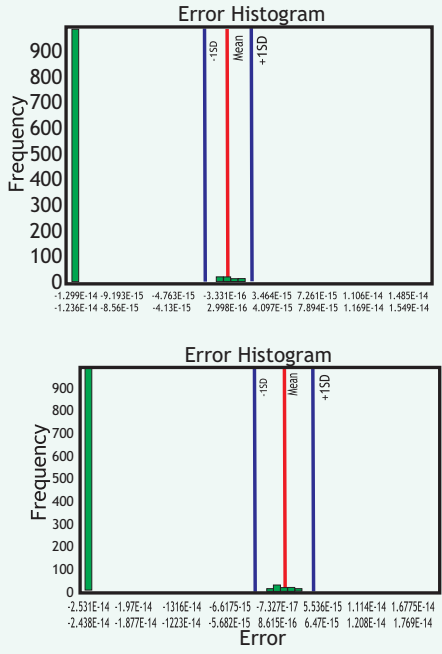

Figure: 10- error histogram for EMS cardiovascular clients o day after:b-LLR, d- DLLR
Tables 5-9. We built and evaluated 5 models for each output.

The MLP type ANNS and linear regression were compared to predicate PM2.5 in Chile. Results showed that ANNS method had the best results for modeling (Perez \& Gramsch, 2000). However, the number of cardiovascular and respiratory EMS clients (Lago, 1, 3,5, and 7 days) was our target. Back- propagation ANN and GA were applied to predict the emergency conditions of Barzilai Medical Center, Israel. GA was used to search network configuration to prevent overtraining. They investigated T, barometric pressure, $\mathrm{RH}, \mathrm{SO}_{2}$, and $\mathrm{NOx}$ as the most important variables in predicting respiratory EMS visits. They evaluated their model with an error of about \%12 (Bibi et al., 2002). We also used the genetic algorithm, but we applied it to find the best combination of the inputs and used the Gamma test to find the threshold limit in the training phase of ANNs. The different MSEs for each technique have been shown in Tables 5-9. As a conclusion, we considered the most effective variables in forecasting our target and then excluded the less effective input variables. Furthermore, sufficient data (6oo out of 1096) and the best Pmax (10) were used in the ANNs training phase. It should be noted that the MSE for threshold value (Table6-8), sufficient data for training ANNs and Pmax were calculated by the Gamma test and the M-test (Fig.2 and 3). In this way, the trial and error for model construction were reduced. Therefore, we improved the accuracy and simplified our modeling.

In the current study, the 3 -day moving average for each input was investigated. However, three standard multilayer perceptron (MLP) models, i.e. time- lagged feed-forward, recurrent ANNs, and Bayesian ANNs were developed for ground-level ozone concentration 
forecasting in Hamilton, Canada. The results showed that all of these models could effectively forecast the ozone concentration (Solaiman, Coulibaly et al., 2008). The applicability of autoregressive integrated moving average (ARIMA), multilinear regression (MLR), and ANNS separately and in combination for air quality forecasting in emergency situations was compared. According to the results, a combination of the ARIMA-ANNS model was more accurate for forecasting pre-emergency air pollution episodes (Díaz-Robles et al., 2008). In developed an MLP ANN with 9 meteorological items and 9 air pollutants for predicting ozone in Istanbul, Turkey and compared its performance with nonlinear regression but found no significant difference between the two methods (Inal, 2010). Three types of feed-forward ANNS were applied to predict $\mathrm{O}_{3}$ with 8 inputs $\left(\mathrm{CO}, \mathrm{NO}_{2}, \mathrm{O}_{3}, \mathrm{~T}, \mathrm{RH}\right.$, and WS). In this study, the GA was used in order to search the number of neurons and activation functions in hidden layers (Pires et al., 2012). Liao et al., (2012). An integrated ANN with case-based reasoning and GA investigated to develop an environmental emergency support system for planning in on-site environmental emergency response (Liao et al., 2012). GT and ANN methods investigated for simulation of global solar radiation in Nigeria. These methods are fitted for other locations(Asagha et al., 2014). Using the winGamma software for optimization of ANN inputs, showed that this method could improve the accuracy (Vo et al., 2013). In most of the above-mentioned studies, the ANN and or LRR were applied to forecast the air pollution and its health consequences in emergency conditions (except for one of them in where the flow rate was its target). Based on experiences and recommendations provided in these studies and other resources, we designed and implemented our research. Preprocessing and optimization will help to solve the main challenge of ANNs to find an "input set" due to the "black box" nature of ANNs (Vo et al., 2013). In the present study, the win Gamma software package was employed for these purposes to discover the best combination of input variables as well as to find the threshold value and criteria for building, running, and testing a data model. In addition, we used the Gamma statistic for calculating the target MSE and applied it for model training and testing to prevent overtraining of the ANN technique. The health consequences of air pollution episodes are delayed and do not occur only on the observed days .So, the 3-day moving average of input variables was added to our dataset. In addition, we assessed their outcomes $0,1,3,5$, and 7 days after observing them.

As mentioned earlier, we applied the LLR, DLLR, and $A_{N N}, A N N_{2}, A N N_{3}$ models. The LLR and DLLR are fast to construct and quite fast to execute predictive tools. However, LLR and DLLR do not generalize well to parts of the input space for which training data is sparse, and if there is a limited number of data points and there is a wide space between them in the vicinity of the query, LLR and DLLR will not be effective if the underlying function is truly non-linear (Jones, 1998).

Standard normalization was used for all 25 input sets and then 9 anomaly input sets were eliminated using GA. Moreover, we optimized the number of neighbors (Pmax..=10) and found the threshold value for filtering the local eigenvectors. By these preprocessing approaches, the MSE 3.5E-30, $1.2 \mathrm{E}-29$ for LLR and 7.84E30, 2.5 E-29 for DLLR were calculated (Table $8 \& 9$ ). In this area, our finding is notable and has a good accuracy due to adequacy and also preprocessing of our dataset. It has been found that the nature of climatological variables has nonlinear and delayed effects (Gasparrini et al., 2010, Gasparrini, 2011). Although ANN models take a time to construct, they can make predictions at blinding speeds as compared with local linear regression. As per obtained results, the MSEs were 0.1-0.27, 0.1-0.25, and 0.1-0.26 for ANN1, ANN2and ANN3models, respectively (Table 5-7).

Conclusively, preprocessing of the given available past value of meteorological and air pollutant variables is essential to predict air pollution conditions (Anomaly detection). GT is a valuable preprocessing tool for building a smooth data model regarding the capability of anomaly detection and association rule learning (Dependency modeling).

GA is an appropriate search and optimization technique to find the best combination of meteorological and air pollutant variables for the prediction of health consequences of atmospheric air pollution (Clustering). ANNs, LLR, and DLLR are powerful executing techniques for forecasting air pollution emergency conditions (Regression \& Summarization).

\section{Acknowledgements:}

The authors wish to thank Tehran University of Medical Sciences for their financial support of this study (grant number \#240/812). We also thank Iran Meteorol. Org. (IRIMO), Tehran Air Quality Control Corporation (TAQCC), and Tehran Emergency Medical Services Center (EMS) for providing us with their daily records.

\section{References:}

Anderson, H. R. (2009): Air pollution and mortality: A history. Atmos., Environ., 43(1): 142-152.

Asagha, E.N., Udo, S.O. \& Echi, I.M. (2014): Modeling and Simulation of Global Solar Radiation in Warri, Nigeria Using Gamma Test and Artificial Neural Network Algorithms. Int. J. Innov. Res. Develop., 3(6):366-374.

Atash, F. (2007): The deterioration of urban environments in developing countries: Mitigating the air pollution crisis in Tehran, Iran. Cities, 24(6):399-409.

Bernard, S.M., Samet, J. M., Grambsch, A., Ebi, K.L. \& Romieu, I. (2001): The potential impacts of climate variability and change on air pollution-related health effects in the United States. Environ. Health Perspec., 109(S-2):199-209.

Bibi, H., Nutman, A. Shoseyov, D., Shalom, M., Peled, R., Kivity, S. Nutman, J. (2002): Prediction of emergency department 
visits for respiratory symptoms using an artificial neural network. Chest Journal., 122(5):1627-1632.

Brunekreef, B. \& Holgate, S.T. (2002): Air pollution and health. The Lancet, 360(9341): 1233-1242.

de Gennaro, G., Trizio, L., Di Gilio, A., Pey, J., Pérez, N., Cusack, M., Alastuey, A. \& Querol, X. (2013): Neural network model for the prediction of PMio daily concentrations in two sites in the Western Mediterranean. Sci.Total Environ., 463-464:875-883.

Demuth, H. \& Beale, M. (2002): Neural network toolbox for use with MATLAB in The MathWorks, Inc. 4th.

Díaz-Robles, L.A., Ortega, J.C., Fu, J. S., Reed, G.D., Chow, J.C., Watson, J.G. \& Moncada-Herrera, J.A. (2008): A hybrid ARIMA and artificial neural networks model to forecast particulate matter in urban areas: The case of Temuco, Chile. Atmosph.Environ., 42(35): 8331-8340.

Durrant, P. J. (2001): A non-linear data analysis and modelling tool with applications to flood prediction. Doctor of Philosophy, Cardiff University.

Elliott, P., Shaddick, G. Wakef ield, J.C., Hoogh, C.D. \& Briggs, D.J. (2007): Long-term associations of outdoor air pollution with mortality in Great Britain. Thorax, 62(12): 1088-1094.

Gardner, M. \& Dorling, S. (1998): Artificial neural networks (the multilayer perceptron)a review of applications in the atmospheric sciences. Atmos. Environ., 32(14):2627-2636.

Gasparrini, A. (2011). Distributed Lag Linear and Non-Linear Models in R: The Package dlnm. J. Statis. Software, 43(8):1-20.

Gasparrini, A., Armstrong, B. Kenward, M.G. (2010): Distributed lag non-linear models. Statis. Med., 29(21):2224-2234.

Goyal, P., Chan, A.T., Jaiswal, N. (2006): Statistical models for the prediction of respirable suspended particulate matter in urban cities. Atmos. Environ., 40(11): 2068-2077.

Grell, G. \& Baklanov, A. (2011): Integrated modeling for forecasting weather and air quality: A call for fully coupled approaches. Atmos.Environ., 45(38): 6845-6851.

Grivas, G. \& Chaloulakou, A. (2006): Artificial neural network models for prediction of PMio hourly concentrations, in the Greater Area of Athens, Greece. Atmos.Environ., 40(7): 12161229.

Hoek, G., Beelen, R., de Hoogh, K. Vienneau, D., Gulliver, J., Fischer, P. \& Briggs, D. (2008): A review of land-use regression models to assess spatial variation of outdoor air pollution." Atmos.Environ., 42(33): 7561-7578.

Holland, J.H. (1975): Adaptation in natural and artificial systems: An introductory analysis with applications to biology, control, and artificial intelligence. Oxford, England, U Michigan Press.

Inal, F. (2010): Artificial Neural Network Prediction of Tropospheric Ozone Concentrations in Istanbul, Turkey. CLEAN-Soil, Air, Water, 38(10): 897-908.

IITM (Indian Institute of Tropical Meteorology) (2015): " Extreme Weather \& Pollution Events: Global\& National", Environmental Information System Center, Acid Rain and Atmospheric Pollution(ARAP), Min. Environ., Forest \& Climate Change, Govt. of India.

Jerrett, M., Arain, A., Kanaroglou, P., Beckerman, B., Potoglou, D., Sahsuvaroglu, T., Morrison, J. \& Giovis, C. (2004): A review and evaluation of intraurban air pollution exposure models. . JExpo. Anal. Environ. Epidemiol., 15(2): 185-204.
Jones, A. (1998): The WinGamma User Guide. Copyright: University of Wales, Cardiff 2001

Liao, Z., Mao, X., Hannam, P.M. \& Zhao, T. (2012): Adaptation methodology of CBR for environmental emergency preparedness system based on an Improved Genetic Algorithm. Expert Sys. Appl., 39(8): 7029-7040.

Liao, Z., Wang, B., Xia, X. \& Hannam, P.M. (2012): Environmental emergency decision support system based on Artificial Neural Network. Safety Sci., 50(1):150-163.

Moustris, K.P., Ziomas, I.C. \& Paliatsos, A.G. (2009):3-Day-Ahead Forecasting of Regional Pollution Index for the Pollutants $\mathrm{NO}_{2}, \mathrm{CO}, \mathrm{SO}_{2}$, and $\mathrm{O}_{3}$ Using Artificial Neural Networks in Athens, Greece. Water Air Soil Poll., 209(1-4):29-43.

Naddafi, K., Hassanvand, M.S., Yunesian, M., Momeniha, F., Nabizadeh, R., Faridi S. \& Gholampour, A. (2012): Health impact assessment of air pollution in megacity of Tehran, Iran. Iran. J. Environ. Health Sci. Eng., 9(1): 28.

Nemery, B., Hoet, P.H.M. \& Nemmar, A. (2001): The Meuse Valley fog of 1930: an air pollution disaster. The Lancet, 357(9257): 704-708.

Noori, R., Karbassi, A.R., Moghaddamnia, A., Han, D., ZokaeiAshtiani, M.H., Farokhnia, A. \& Gousheh, M.G. (2011): Assessment of input variables determination on the SVM model performance using PCA, Gamma test, and forward selection techniques for monthly stream flow prediction. $L$. Hydrol., 401(3-4):177-189.

Perez, P. \& Gramsch, E. (200o): Forecasting hourly PM2.5 in Santiago de Chile with emphasis on night episodes. Atmos.Environ., 124(A): 22-27.

Pires, J.C., Goncalves, B., Azevedo, F.G., Carneiro, A.P. Rego, N., Assembleia, A.J., Lima, J.F., Silva, P.A., Alves, C.A. \& Martins, F.G. (2012): Optimization of artif icial neural network models through genetic algorithms for surface ozone concentration forecasting.Environ. Sci. Pollut. Res. Int., 19(8):3228-3234.

Malhotra, R., Singh, S. \& Singh Y. (2011): Genetic Algorithms: Concepts, Design for Optimization of Process Controllers. Canad. Centre Sci.Edu., 4(2):16.

Sof ianopoulou, E., Rushton, S.P., Diggle, P.J. \& Pless-Mulloli, T. (2013): Association between respiratory prescribing, air pollution and deprivation, in primary health care. J. Public Health, 35(4):502-509.

Solaiman, T.A., Coulibaly, P. \& Kanaroglou, P. (2008): Groundlevel ozone forecasting using data-driven methods. Air Qual. Atmos. Hlth., 1(4): 179-193.

Solaiman, T.A., Coulibaly, P. \& Kanaroglou, P. (2009): Groundlevel ozone forecasting using data-driven methods. Air Qual. Atmos. Hlth., 1(4): 179-193.

Vanos, J.K., Cakmak, S., Kalkstein, L.S. \& Yagouti, A. (2015): Association of weather and air pollution interactions on daily mortality in 12 Canadian cities. Air Qual. Atmos. Hlth., 8(3): 307-320.

Vo, N., Shi, H. \& Szajman, J. (2013): Optimisation to ANN Inputs in Automated Property Valuation Model with Encog 3 and winGamma. Appl. Mech. Mat., 462-463: 1081-1086.

Zannetti, P. (2013): Air Pollution Modeling: Theories, computational methods and available software. Springer Science \& Business Media. 